\title{
A systematic review of the prevalence and risk factors for adverse drug reactions in the elderly in the acute care setting
}

This article was published in the following Dove Press journal:

Clinical Interventions in Aging

I December 2014

Number of times this article has been viewed

\author{
Tariq M Alhawassi ${ }^{1,2}$ \\ Ines Krass' \\ Beata $\vee$ Bajorek ${ }^{3,4}$ \\ Lisa G Pont ${ }^{5}$
}

'Faculty of Pharmacy, University of Sydney, Sydney, NSW, Australia; ${ }^{2}$ College of Pharmacy, King Saud University, Riyadh, Saudi Arabia; ${ }^{3}$ Graduate School of Health Pharmacy, University of Technology Sydney, ${ }^{4}$ Pharmacy Department, Royal North Shore Hospital, ${ }^{5}$ Sydney Nursing School, University of Sydney, Sydney, NSW, Australia
Correspondence: Lisa G Pont M02 - Sydney Nursing School, The University of Sydney, NSW 2006, Australia

Tel +6I 2 935। 0563

Fax +6I 2935 I 0779

Email lisa.pont@sydney.edu.au
Abstract: Adverse drug reactions (ADRs) are an important health issue. While prevalence and risk factors associated with ADRs in the general adult population have been well documented, much less is known about ADRs in the elderly population. The aim of this study was to review the published literature to estimate the prevalence of ADRs in the elderly in the acute care setting and identify factors associated with an increased risk of an ADR in the elderly. A systematic review of studies published between 2003 and 2013 was conducted in the Cochrane Database of Systematic Reviews, EMBASE, Google Scholar and MEDLINE. Key search terms included: "adverse drug reactions", "adverse effects", "elderly patients and hospital admission", "drug therapy”, "drug adverse effects", "drug related”, “aged”, "older patients”, "geriatric”, "hospitalization", and "emergency admissions". For inclusion in the review, studies had to focus on ADRs in the elderly and had to include an explicit definition of what was considered an ADR and/or an explicit assessment of causality, and a clear description of the method used for ADR identification, and had to describe factors associated with an increased risk of an ADR. Fourteen hospital-based observational studies exploring ADRs in the elderly in the acute care setting were eligible for inclusion in this review. The mean prevalence of ADRs in the elderly in the studies included in this review was $11.0 \%$ (95\% confidence interval [CI]: 5.1\%-16.8\%). The median prevalence of ADRs leading to hospitalization was 10.0\% (95\% CI: $7.2 \%-12.8 \%)$, while the prevalence of ADRs occurring during hospitalization was 11.5\% (95\% CI: 0\%-27.7\%). There was wide variation in the overall ADR prevalence, from 5.8\% to $46.3 \%$. Female sex, increased comorbid complexity, and increased number of medications were all significantly associated with an increased risk of an ADR. Retrospective studies and those relying on identification by the usual treating team reported lower prevalence rates. From this review, we can conclude that ADRs constitute a significant health issue for the elderly in the acute care setting. While there was wide variation in the prevalence of ADRs in the elderly, based on the findings of this study, at least one in ten elderly patients will experience an ADR leading to or during their hospital stay. Older female patients and those with multiple comorbidities and medications appear to be at the highest risk of an ADR in the acute care setting.

Keywords: drug utilization, hospital

\section{Introduction}

Pharmacotherapy plays an important role in the maintenance of health. Many conditions rely on medication to manage symptoms, slow disease progression, or to prevent the development of future illnesses. While medications may provide considerable benefit in the maintenance of health, all medications also have a risk of adverse or unwanted effects, and evaluating the risk/balance benefit associated with use of a particular medication is a critical step in the decision to use pharmacotherapy. Adverse drug 
reactions (ADRs) are adverse or unintended effects associated with medication and have been defined by the World Health Organization (WHO) as "noxious and unintended responses to drugs occurring at doses normally used in man for the prophylaxis, diagnosis or therapy of disease, or for modification of physiological function". ${ }^{1}$ ADRs are an important component of adverse drug events, which encompass medication-related harm associated with ADRs as well as that secondary to errors. ${ }^{2}$

ADRs have a significant impact on heath, with between $5 \%$ and $7 \%$ of all hospitalizations being due to an ADR and with a further $10 \%$ to $20 \%$ of all hospitalized patients experiencing an ADR during their hospital admission..$^{2-4}$ Between $3 \%$ and $6 \%$ of ADRs are fatal or have serious consequences, with an estimated 140,000 fatalities secondary to ADRs occurring annually in the USA..$^{5-8}$ The estimated impact on hospital costs exceeds $\$ 30$ billion, or $5 \%$ of total hospital running costs per annum..$^{8-10}$

While the prevalence and impact of ADRs has been well studied in the general adult population, ${ }^{11}$ much less is known about ADRs in the elderly. Older persons experience an increased disease burden and a corresponding increase in medication utilization, which has been well documented. ${ }^{12}$ With this increased medication use and health complexity, there is a corresponding increased risk of ADRs. As well as increases in medication number and comorbid conditions, there are a variety of age-related physiological changes affecting the pharmacokinetics and pharmacodynamics of medications, which may further increase the risk of ADRs in older persons.

Despite the potential increased risk of ADRs in older persons, little is known about the prevalence of ADRs and the associated risk factors in the elderly population. The aim of this review was to determine the prevalence of ADRs, leading to and during admission, in the elderly, to identify characteristics associated with an increased ADR risk in elderly persons and to examine how the prevalence varies with different study designs and identification strategies in the acute care setting.

\section{Methods}

\section{Data sources and study selection}

An electronic search of the Cochrane Database of Systematic Reviews, EMBASE, Google Scholar, and MEDLINE for studies published between 2003 and 2013 was conducted. Medical Subject Headings terminology was used where possible (in MEDLINE and Cochrane) and keywords used in those databases not using Medical Subject Headings terminology (EMBASE and Google scholar). The terms used were: "adverse drug reactions", "adverse effects", and "elderly patients" and "hospital admission". Additional search terms used included: "drug therapy", "drug adverse effects", "drug related", “aged", "older patients", "geriatric”, "hospitalization", and "emergency admissions".

All titles and abstracts of identified studies were examined for potential relevance. Reference lists of identified studies were reviewed to locate other relevant original studies. Eligible study titles and abstracts were screened for relevance, and a manual cross-reference search of the relevant papers was performed to identify additional articles. Two independent reviewers assessed each study, and final consensus was reached regarding inclusion of each study before data extraction.

\section{Inclusion criteria}

Studies were included in the review if they were published in English with a primary aim to assess ADR prevalence, either leading to hospitalization or during admission, in the elderly ( $\geq 65$ years) in an acute care setting, using observational methods. For inclusion in the review, studies had to include an explicit definition of what was considered an ADR and/or an explicit assessment of causality, as well as a clear description of the method used for ADR identification, and had to explore factors associated with an increased risk of an ADR.

\section{Exclusion criteria}

Studies lacking a precise ADR definition or those without explicit causality assessment criteria, those that focused on ADRs secondary to a specific medication or specific ADR, and studies that looked at ADR-related hospital readmissions were excluded from the review. Studies that did not report ADR prevalence data or allow calculation of prevalence were also excluded.

\section{Data extraction, synthesis, and analysis}

Data were extracted, including ADR definition, identification method, risk factors, and prevalence, using a customdesigned data extraction form. Where the prevalence was not directly reported in the original publication, it was calculated as the number of patients identified with an ADR out of all included patients. Extracted data were entered into Microsoft Excel ${ }^{\mathrm{TM}}$ for descriptive data analysis. Overall median ADR prevalence as well as median prevalence of ADRs, leading to hospitalization and those occurring during inpatient stay, were calculated along with the corresponding 95\% confidence intervals (CIs). The quality of included studies was determined using the Strengthening 
the Reporting of Observational Studies in Epidemiology (STROBE) criteria for observational studies. ${ }^{13}$ The protocol for this systematic review was registered on the International Prospective Register of Systematic Reviews (PROSPERO) international prospective register of systematic reviews (CRD42013006827). ${ }^{14}$

\section{Results}

Fourteen hospital-based observational studies exploring ADRs in the elderly in acute care settings were identified and eligible for inclusion in this review (Figure 1). The size and design of the studies varied from large retrospective observational administrative data cohorts to smaller prospective studies in the clinical setting. The included studies ranged in size from a small prospective study of 80 patients presenting to Emergency in Belgium ${ }^{15}$ to a large populationbased cohort of 64,446 Canadian hospital admissions. ${ }^{16}$ The majority of studies were conducted in Europe $(n=10)$, with two studies from Asia and two from North America. Characteristics and a summary of the included studies are presented in Table 1.

\section{Prevalence of ADRs in the elderly}

The mean prevalence of ADRs in the elderly in the studies included in this review was $11.0 \%$ (95\% CI: 5.1-16.8).

The median prevalence of ADRs leading to hospitalization

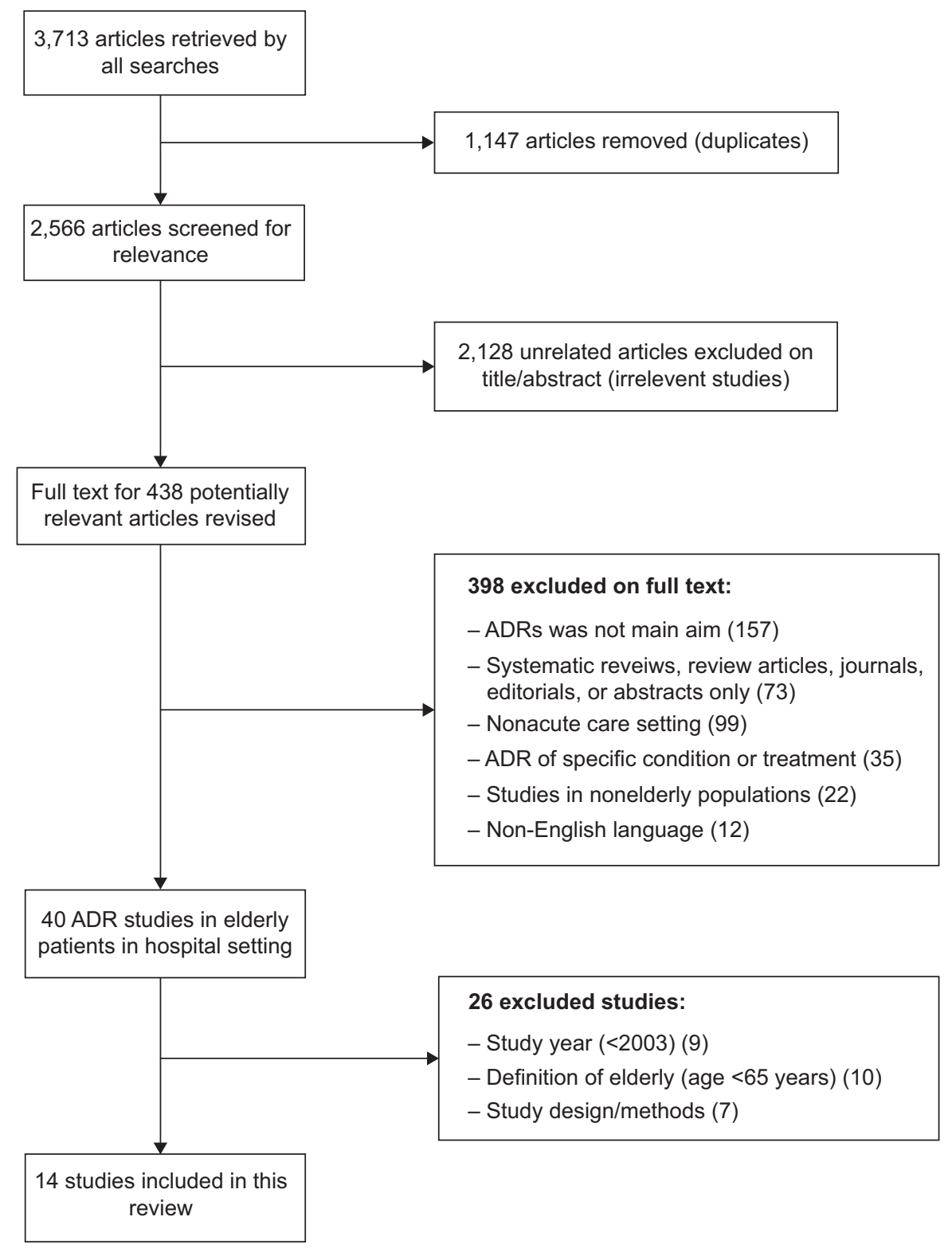

Figure I Search strategy and identification of included studies. Abbreviation: ADR, adverse drug reaction. 


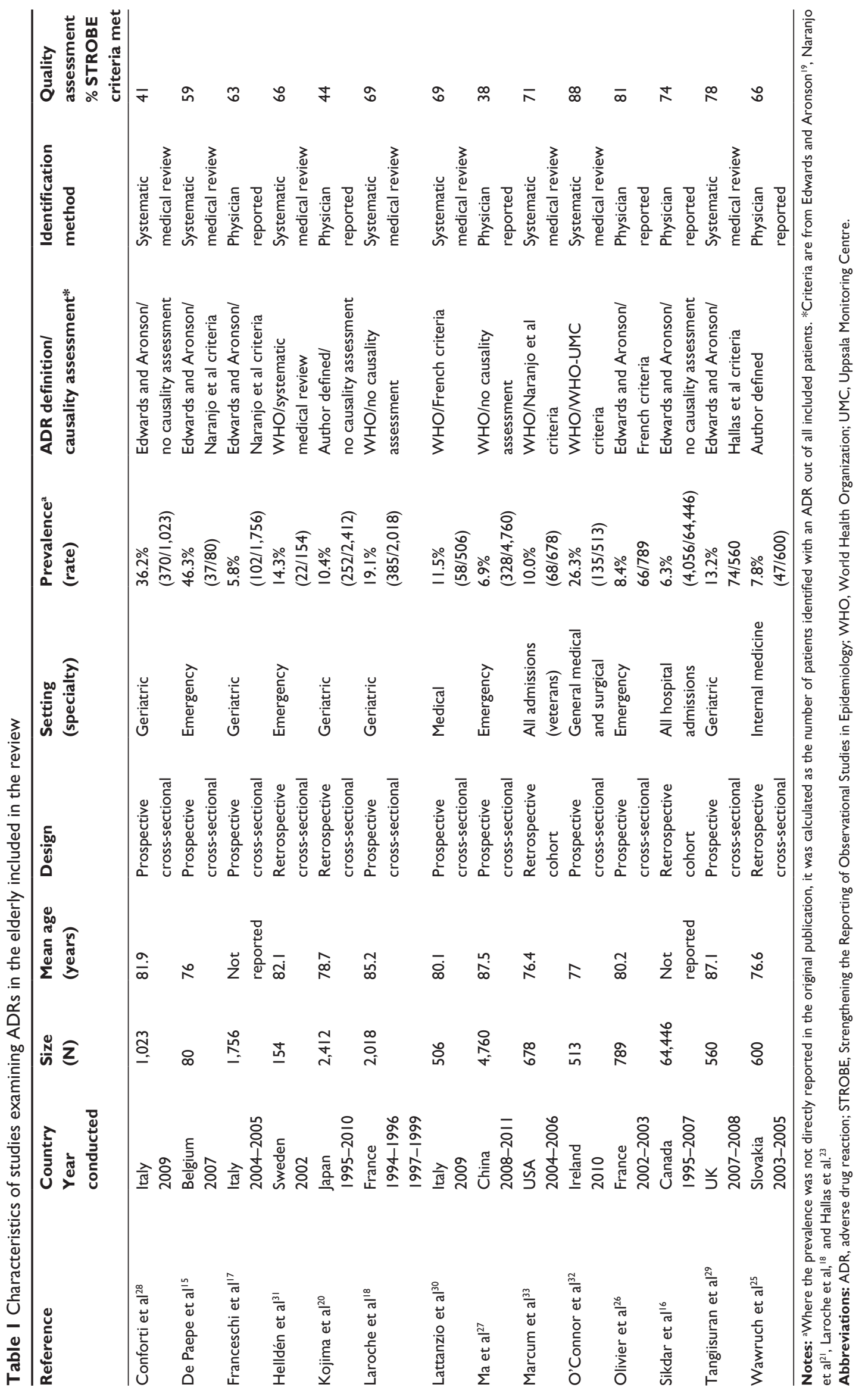


was $10.0 \%$ (95\% CI: $7.2 \%-12.8 \%$ ), while the prevalence of ADRs occurring during hospitalization was $11.5 \%$ (95\% CI: $0 \%-27.7 \%$ ). There was wide variation in the overall ADR prevalence reported in the studies in this review, from a prevalence of $5.8 \%$ in 1,756 older Italian patients ${ }^{17}$ to $46.3 \%$ in a smaller Belgian study. ${ }^{15}$ Prospective studies were associated with higher prevalence rates than were retrospective studies (Table 1). Larger studies generally tended to report higher prevalence rates, with the exception of the French study by Laroche et al. ${ }^{18}$

\section{Defining and identifying ADRs}

The use of a predefined definition of ADRs or explicit assessment of causality was an inclusion criterion for this analysis. In general, there was consensus regarding the definitions of ADRs. The majority of the studies $(n=8)$ used either the WHO definition $^{1}$ or that proposed by Edwards and Aronson:

An appreciably harmful or unpleasant reaction, resulting from an intervention related to the use of a medicinal product, which predicts hazard from future administration and warrants prevention or specific treatment, or alteration of the dosage regimen or withdrawal of the product. ${ }^{19}$

Only two studies used custom predefined definitions. ${ }^{20,21}$ Half $(n=7)$ of the studies included in this review described an explicit causality assessment. The most commonly used criteria were the Naranjo et al criteria, ${ }^{21}$ used by three studies. Two studies used the French method of causality assessment, ${ }^{22}$ one used the Hallas et al criteria ${ }^{23}$ and one the WHO-Uppsala Monitoring Committee criteria $^{25}$ (Table 1). There did not appear to be a relationship between the use of explicit casualty criteria and the ADR prevalence reported.

A number of methods were used to identify ADRs in the included studies. Most studies $(n=8)$ used a systematic medical review where a dedicated study team identified potential ADRs. A number of studies $(n=6)$ relied upon physician reporting, where ADRs were identified as part of usual care. ${ }^{16,17,20-27}$ Four prospective studies relied on identification of potential ADRs by physician reporting, ${ }^{17,21,26,28}$ while the remaining two studies were retrospective cohort studies using administrative datasets and identified potential ADRs retrospectively from the medical record. ${ }^{16,20}$ The majority of studies $(n=10)$ outlined separate processes in addition to usual care for the identification of potential ADRs. ${ }^{15,17,18,27-33}$ These usually involved identification of a potential ADR by a member or members of the research team and then systematic medical review of the potential ADR via expert panel review.
Only one study provided explicit detail regarding the criteria used to screen patients for potential ADRs. ${ }^{33}$

\section{Medications associated with ADRs}

There was considerable variation in the medications involved in the ADRs reported in the studies. Many studies found cardiovascular medications to be commonly associated with ADRs in the elderly, in particular antihypertensive and antithrombotic medications. ${ }^{16-18,26-28}$ Other implicated medications included antibiotics, ${ }^{26,27}$ nonsteroidal antiinflammatories, ${ }^{17,27}$ and antidiabetic agents. ${ }^{29}$

\section{ADR-associated risk factors in the elderly}

A number of risk factors associated with ADR in the elderly in acute care were identified in this review. These have been categorized as patient factors, disease factors, medication factors, or other factors (Table 2).

While all studies were conducted on populations aged 65 years or older, two studies found increasing age to be an independent ADR risk factor. ${ }^{16,32}$ Female sex was also consistently identified as a risk factor in the majority of studies. ${ }^{15,16,28,30}$

Increasing medical complexity, both in terms of increasing comorbid burden ${ }^{16,25,29}$ and increased number of

Table 2 Identified risk factors associated with ADR in the elderly

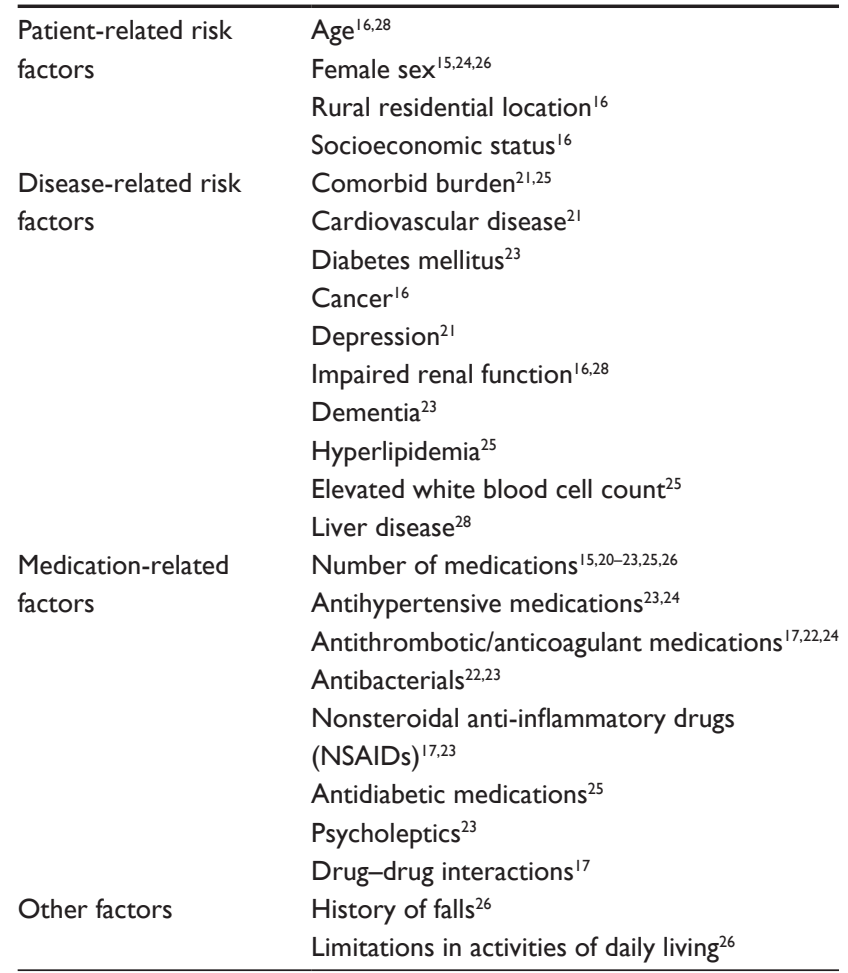

Abbreviation: ADR, adverse drug reaction. 
medications, ${ }^{15,18,20,25-27,30,32}$ were both reported to be associated with an increased ADR risk, in a number of studies.

\section{Discussion}

The findings of this systematic review indicate that ADRs constitute a significant health care issue for the elderly in the acute care setting, suggesting that more than one in every ten older patients in the hospital setting experience an ADR, either leading to or during their hospital admission.

Previous work has estimated that up to $17 \%$ of all adult patients admitted to hospital experience an ADR either leading to or during their hospital admission. ${ }^{34}$ A large meta-analysis suggested that the elderly had a four times higher risk of an ADR than did the general adult population; however, this meta-analysis was not focused specifically on the elderly and analyzed the over-65 age group as a subgroup analysis. ${ }^{34}$ There was wide variability in the ADR prevalence rates reported in this review, ranging from just over $5 \%$ to almost $50 \%$ of elderly patients. Heterogeneity in how ADRs are defined and identified are likely to account for much of this variability. In our review, higher prevalence rates tended to be found in larger studies and those using a prospective design.

The majority of studies in the analyses used either the WHO definition ${ }^{1}$ of an ADR or that proposed by Edwards and Aronson. ${ }^{19}$ While these definitions have fundamental similarities in terms of harm associated with use of pharmacological products, distinct differences exist in terms of what is considered an ADR under each definition, especially with respect to minor ADRs. The WHO definition does not specify a level of harm in the initial identification of a potential ADR and may capture more minor ADRs, which while having a significant impact on quality of life, do not lead to serious outcomes such as death, disability, or hospitalization. Another difference between the two definitions is the focus in the WHO description on a "drug" as the causative agent, rather than a "medicinal product", as specified in the Edwards and Aronson definition. The broader Edwards and Aaronson definition may be interpreted to encourage the inclusion of reactions associated with complementary and herbal preparations and may thus affect the ADR prevalence rates reported. In general, in the studies included in this review, those using the WHO definition reported a narrower prevalence range than did those using the Edwards and Aaronson definition. However, ADR definition is only one factor that will contribute to differences reported between the studies with respect to prevalence.
Differences in the methods used to detect ADRs may be another factor contributing to the wide range of prevalence rates reported. A number of studies relied on the identification of ADRs during routine care; however, underidentification ${ }^{35,36}$ and underreporting ${ }^{37-39}$ of ADRs during routine care in the acute care setting has been well documented in the literature, and such methods are likely to underestimate the true prevalence of ADRs. This may have been the case in the Sikdar et al study, which relied on identification of ADRs during routine clinical care and then further relied on these episodes being accurately coded in the administrative dataset from which they were identified. ${ }^{16}$ While this study had the largest sample size in the review $(n=64,446)$, the reliance on ADR identification during routine clinical care would have excluded undiagnosed ADRs and may have contributed toward one of the lowest reported prevalence rates among the included studies. It is important to note that retrospective studies rely on routine detection of ADRs as captured in medical records and case notes and may underrepresent the true ADR prevalence. Two other studies that also relied on routine detection of ADRs also reported relatively low prevalence rates, ${ }^{25,26}$ further supporting concerns regarding the possible underdetection of ADRs during routine care.

While there was variation in the medications associated with ADRs in the elderly across the 14 studies included in this review, there were medications common to a number of studies, which may raise concerns regarding their safe use in the elderly. Cardiovascular medications were associated with ADRs in the elderly in a number of the studies in this review. ${ }^{16-18,26-28}$ Specific cardiovascular medications identified included antihypertensives, diuretics, and angiotensin-converting enzyme inhibitors, all of which have been associated with a number of issues, such as falls and electrolyte disturbances, in the elderly; ${ }^{40,41}$ further investigation may be required to fully understand their role in ADR risk.

Despite the heterogeneity among the studies, there was considerable consistency in the risk factors associated with ADRs in elderly inpatients. Female sex was consistently identified as a risk factor; however, it was unclear whether this related to an increased ADR risk or an increased use of medications. ${ }^{15,16,28,30}$ Advanced age was found a risk factor by two of the studies in this review studies. ${ }^{16}$ Increased age was not only associated with increased frailty and physiological changes resulting in changed pharmacokinetics and dynamics, but also, with increased medical complexity, disease burden and medication use. Both increased disease burden and medication number were identified as independent risk 
factors for ADRs in the elderly; however, teasing out the complex relationship between aging, disease burden, medication use, and the risk of an ADR remains problematic. While disease burden was identified as a risk factor in a number of studies, ${ }^{16,25,29}$ there was great diversity regarding the role of specific conditions, making it difficult to fully understand the contribution of specific conditions to the risk of ADRs in the elderly.

A main strength of this study was the strict inclusion criteria applied regarding ADR definitions and identification. The review was limited to studies that included explicit criteria for both what was considered an ADR and how the ADRs were assessed in terms of causality. In addition, all studies required clear description of the method applied in the identification of the ADRs. The review was limited to observational studies in the acute care setting that focused specifically on ADRs in patients aged 65 years and older, in an attempt to minimize the heterogeneity among included studies. However, despite the strict inclusion criteria, considerable heterogeneity was found among the 14 studies included in this review. The quality of included studies varied extensively. While most of the included studies fulfilled more than $50 \%$ of the STROBE criteria and could be considered to have moderate quality, ${ }^{42}$ no study met these criteria completely. Of particular note, only three studies reported a priori sample size calculations. Sample size calculations for crosssectional studies are based upon the expected prevalence of the parameter under investigation, and small sample sizes may not provide adequate precision. In this review, studies with smaller sample sizes tended to have higher prevalence estimates. This heterogeneity limited the ability to pool data and provide summary estimates for ADR prevalence across the total review population.

\section{Conclusion}

ADRs constitute a significant health issue for the elderly in the acute care setting. This review identified that at a conservative estimate, one in ten elderly patients will experience an ADR leading to or during their hospital stay and that older patients with an increased disease burden and using a higher number of medications are at increased risk of an ADR leading to or during their hospital stay. Differences in what is considered an ADR and in the identification of potential ADRs lead to considerable heterogeneity between studies examining ADRs in the elderly. Future research focused on identifying and minimizing the risks of ADRs in the elderly is needed, to aid clinicians in the optimal use of pharmacotherapy in older patients.

\section{Disclosure}

The authors report no conflicts of interest in this work.

\section{References}

1. World Health Organization. International drug monitoring. The role of the hospital. World Health Organ Tech Rep Ser. 1969;425:5-24.

2. Davies EC, Green CF, Mottram DR, Pirmohamed M. Adverse drug reactions in hospitals: a narrative review. Curr Drug Saf. 2007;2(1): 79-87.

3. Pirmohamed M, James S, Meakin S, et al. Adverse drug reactions as cause of admission to hospital: prospective analysis of 18,820 patients. BMJ. 2004;329(7456):15-19.

4. Davies EC, Green CF, Taylor S, Williamson PR, Mottram DR, Pirmohamed M. Adverse drug reactions in hospital in-patients: a prospective analysis of 3,695 patient-episodes. PLoS One. 2009;4(2):e4439.

5. Moore N, Lecointre D, Noblet C, Mabille M. Frequency and cost of serious adverse drug reactions in a department of general medicine. Br J Clin Pharmacol. 1998;45(3):301-308.

6. Wester K, Jönsson AK, Spigset O, Druid H, Hägg S. Incidence of fatal adverse drug reactions: a population based study. Br JClin Pharmacol. 2008;65(4):573-579.

7. Lazarou J, Pomeranz BH, Corey PN. Incidence of adverse drug reactions in hospitalized patients: a meta-analysis of prospective studies. JAMA.1998;279(15):1200-1205.

8. White TJ, Arakelian A, Rho JP. Counting the costs of drug-related adverse events. Pharmacoeconomics. 1999;15(5):445-458.

9. Tangiisuran B, Gozzoli MP, Davies JG, Rajkumar C. Adverse drug reactions in older people. Reviews in Clinical Gerontology. 2010;20(03): 246-259.

10. Patel KJ, Kedia MS, Bajpai D, Mehta SS, Kshirsagar NA, Gogtay NJ. Evaluation of the prevalence and economic burden of adverse drug reactions presenting to the medical emergency department of a tertiary referral centre: a prospective study. BMC Clin Pharmacol. 2007;7:8.

11. Dormann H, Criegee-Rieck M, Neubert A, et al. Lack of awareness of community-acquired adverse drug reactions upon hospital admission: dimensions and consequences of a dilemma. Drug Saf. 2003; 26(5):353-362.

12. Lehnert $\mathrm{T}$, Heider $\mathrm{D}$, Leicht $\mathrm{H}$, et al. Review: health care utilization and costs of elderly persons with multiple chronic conditions. Med Care Res Rev. 2011;68(4):387-420.

13. von Elm E, Altman DG, Egger M, Pocock SJ, Gøtzsche PC, Vandenbroucke JP; STROBE Initiative. The Strengthening the Reporting of Observational Studies in Epidemiology (STROBE) statement: guidelines for reporting observational studies. Lancet. 2007; 370(9596):1453-1457.

14. crd.york.ac.uk/PROSPERO [homepage on the Internet]. PROSPERO international prospective register of systematic reviews. Centre for Reviews and Dissemination, University of York; 2013 [updated November 28, 2013; cited August 31, 2014]. Available from: http://www.crd. york.ac.uk/PROSPERO. Accessed September 13, 2014.

15. De Paepe P, Petrovic M, Outtier L, Van Maele G, Buylaert W. Drug interactions and adverse drug reactions in the older patients admitted to the emergency department. Acta Clin Belg. 2013;68(1):15-21.

16. Sikdar KC, Dowden J, Alaghehbandan R, MacDonald D, Peter P, Gadag V. Adverse drug reactions in elderly hospitalized patients: a 12-year population-based retrospective cohort study. Ann Pharmacother. 2012;46(7-8):960-971.

17. Franceschi M, Scarcelli C, Niro V, et al. Prevalence, clinical features and avoidability of adverse drug reactions as cause of admission to a geriatric unit: a prospective study of 1,756 patients. Drug Saf. 2008;31(6):545-556.

18. Laroche ML, Charmes JP, Nouaille Y, Picard N, Merle L. Is inappropriate medication use a major cause of adverse drug reactions in the elderly? Br J Clin Pharmacol. 2007;63(2):177-186.

19. Edwards IR, Aronson JK. Adverse drug reactions: definitions, diagnosis, and management. Lancet. 2000;356(9237):1255-1259. 
20. Kojima T, Akishita M, Kameyama Y, et al. High risk of adverse drug reactions in elderly patients taking six or more drugs: analysis of inpatient database. Geriatr Gerontol Int. 2012;12(4):761-762.

21. Naranjo CA, Busto U, Sellers EM, et al. A method for estimating the probability of adverse drug reactions. Clin Pharacol Ther. 1981; 30(2):239-245.

22. Bégaud B, Evreux JC, Jouglard J, Lagier G. [Imputation of the unexpected or toxic effects of drugs. Actualization of the method used in France]. Therapie. 1985;40(2):111-118. French.

23. Hallas J, Harvald B, Gram LF, et al. Drug related hospital admissions: the role of definitions and intensity of data collection, and the possibility of prevention. J Intern Med. 1990;228(2):83-90.

24. The Uppsala Monitoring Centre. The Use of the WHO-UMC System for Standardised Case Causality Assessment. Uppsala: Uppsala Monitoring Centre; 2012. Available from: http://who-umc.org/Graphics/26649.pdf. Accessed August 31, 2014.

25. Wawruch M, Zikavska M, Wsolova L, et al. Adverse drug reactions related to hospital admission in Slovak elderly patients. Arch Gerontol Geriatr. 2009;48(2):186-190.

26. Olivier P, Bertrand L, Tubery M, Lauque D, Montastruc JL, LapeyreMestre M. Hospitalizations because of adverse drug reactions in elderly patients admitted through the emergency department: a prospective survey. Drugs Aging. 2009;26(6):475-482.

27. Ma J, Wang Y, Gao M, Meng Q, Liu J. Adverse drug reactions as the cause of emergency department admission of patients aged 80 years and older. Eur J Intern Med. 2012;23(6):e162-e163.

28. Conforti A, Costantini D, Zanetti F, Moretti U, Grezzana M, Leone R. Adverse drug reactions in older patients: an Italian observational prospective hospital study. Drug Healthc Patient Saf. 2012;4:75-80.

29. Tangiisuran B, Davies JG, Wright JE, Rajkumar C. Adverse drug reactions in a population of hospitalized very elderly patients. Drugs Aging. 2012;29(8):669-679.

30. Lattanzio F, Laino I, Pedone C, et al; PharmacosurVeillance in the elderly Care (PVC) Study Group. Geriatric conditions and adverse drug reactions in elderly hospitalized patients. J Am Med Dir Assoc. 2012; 13(2):96-99.

31. Helldén A, Bergman U, von Euler M, Hentschke M, Odar-Cederlöf I, Ohlén G. Adverse drug reactions and impaired renal function in elderly patients admitted to the emergency department: a retrospective study. Drugs Aging. 2009;26(7):595-606.
32. O’Connor MN, Gallagher P, Byrne S, O’Mahony D. Adverse drug reactions in older patients during hospitalisation: are they predictable? Age Ageing. 2012;41(6):771-776.

33. Marcum ZA, Amuan ME, Hanlon JT, et al. Prevalence of unplanned hospitalizations caused by adverse drug reactions in older veterans. J Am Geriatr Soc. 2012;60(1):34-41.

34. Beijer HJ, de Blaey CJ. Hospitalisations caused by adverse drug reactions (ADR): a meta-analysis of observational studies. Pharm World Sci. 2002;24(2):46-54.

35. Kongkaew C, Noyce PR, Ashcroft DM. Hospital admissions associated with adverse drug reactions: a systematic review of prospective observational studies. Ann Pharmacother. 2008;42(7):1017-1025.

36. Field TS, Gurwitz JH, Harrold LR, et al. Strategies for detecting adverse drug events among older persons in the ambulatory setting. J Am Med Inform Assoc. 2004;11(6):492-498.

37. Brvar M, Fokter N, Bunc M, Mozina M. The frequency of adverse drug reaction related admissions according to method of detection, admission urgency and medical department specialty. BMC Clin Pharmacol. 2009;9:8.

38. Gony $\mathrm{M}$, Badie $\mathrm{K}$, Sommet $\mathrm{A}$, et al. Improving adverse drug reaction reporting in hospitals: results of the French Pharmacovigilance in MidiPyrénées region (PharmacoMIP) network 2-year pilot study. Drug Saf. 2010;33(5):409-416.

39. Hazell L, Shakir SA. Under-reporting of adverse drug reactions: a systematic review. Drug Saf. 2006;29(5):385-396.

40. Huang AR, Mallet L, Rochefort CM, Eguale T, Buckeridge DL, Tamblyn R. Medication-related falls in the elderly: causative factors and preventive strategies. Drugs Aging. 2012;29(5):359-376.

41. Budnitz DS, Shehab N, Kegler SR, Richards CL. Medication use leading to emergency department visits for adverse drug events in older adults. Ann Intern Med. 2007;147(11):755-765.

42. Olmos M, Antelo M, Vazquez H, Smecuol E, Mauriño E, Bai JC. Systematic review and meta-analysis of observational studies on the prevalence of fractures in coeliac disease. Dig Liver Dis. 2008;40(1): 46-53.
Clinical Interventions in Aging

\section{Publish your work in this journal}

Clinical Interventions in Aging is an international, peer-reviewed journal focusing on evidence-based reports on the value or lack thereof of treatments intended to prevent or delay the onset of maladaptive correlates of aging in human beings. This journal is indexed on PubMed Central, MedLine,

\section{Dovepress}

CAS, Scopus and the Elsevier Bibliographic databases. The manuscript management system is completely online and includes a very quick and fair peer-review system, which is all easy to use. Visit http://www.dovepress. com/testimonials.php to read real quotes from published authors. 